\title{
Laser-Assisted Frenectomy Using 980nm Diode Laser
}

\author{
Tahrir N Aldelaimi ${ }^{*}$ and Ali S Mahmood ${ }^{2}$ \\ ${ }^{1}$ Department of Oral \& Maxillofacial Surgery, College of Dentistry, Anbar University, Iraq \\ ${ }^{2}$ Biomedical Department, Institute of laser for post-graduate studies, University of Bhagdad, Iraq
}

\begin{abstract}
Received: October 14, 2014; Accepted: November 05, 2014; Published: November 15, 2014
*Corresponding author: Tahir N Aldelaimi, Department of Oral \& Maxillofacial Surgery, College of Dentistry, Anbar University, Iraq, Tel: 009647814050913; E-mail: tahrir_aldelaimi@yahoo.com
\end{abstract}

\begin{abstract}
A frenum is a fold of tissue or muscle connecting the lips, cheek or tongue to the jawbone. Their high attachment may cause orthodontic problem (Central diastima), prosthodontic problems (Poor retention of denture), periodontic problems (gingival recession and inflammation) and speech problem (tongue tied in ligual frenum). A frenectomy is the removal of one of these folds of tissue and it is a common procedure in the specialty of maxillofacial surgery. Laser-assisted frenectomy is a much more comfortable for the patient because it means that no scalpel or stitches are required. This clinical study was carried out at department of Maxillofacial Surgery, Ramadi Teaching Hospital; 25 patients including 16 (64\%) male and $9(36 \%)$ female with age range from 3 months to 18 years old with mean age 6.5 years old. Chirolas 20W diode laser emitting at $980 \mathrm{~nm}$; the laser is delivered through a fiber optic $400 \mu \mathrm{m}$. Beam profile is a single-mode Gaussian $\left(\mathrm{TEM}_{00}\right)$, with a spot size $(0.9 \mathrm{~mm})$ was used to performed laser-assisted frenectomy. Our preliminary clinical findings include sufficient hemostasis, coagulation properties and precise incision margin with all of the surgical procedure. The postoperative advantages, i.e., lack of swelling, bleeding, pain or, scar tissue formation, the good wound healing and overall satisfaction were observed in the clinical application of laser-assisted frenectomy. The clinical application of the diode $(980 \mathrm{~nm})$ laser in oral and maxillofacial surgical procedures proved to be of beneficial effect for daily practice, it can be considered practical, effective, easy to used, offers a safe, acceptable, and impressive alternative for conventional techniques of frenectomy.
\end{abstract}

Keywords: Laser surgery; Frenectomy; Labial frenum; Lingual frenum; Laser application; Oral surgery

\section{Introduction}

Since the first report on laser radiation by Maiman (1960), many potential fields for its application have been investigated $[1,2]$. Among these, medical laser surgery certainly belongs to the most significant advances. Various kinds of lasers have already become irreplaceable tools of modern medicine. Although clinical applications were first limited to ophthalmology and dentistry, the other fields of medical laser treatment have meanwhile considerably widened. Soon after laser invention was demonstrated, researchers began to examine Maiman's vision of the laser as a useful instrument for medicine. Their efforts laid the foundation for the present clinical use of lasers in medicine [3-5]. The specialty of maxillofacial surgery has benefited from the use of lasers since the mid-1960s, with the first documented use of a laser in 1977; lasers are quickly becoming the standard of care for many surgical procedures, given the advantages of improved precision, visualization, and reduced discomfort and lasers are versatile and valuable devices in various disciplines of dentistry-prosthetics, periodontics, pedodontics, endodontics, implantology, cosmetic and operative dentistry, and oral and maxillofacial surgery $[3,6]$. Lasers are becoming more commonplace and even routine, either as adjunctive treatment methodologies or as stand-alone additions to the dental armamentarium. The growing number of dental laser practitioners, propelled by the increasing body of evidence concerning the safe, effective, and appropriate use of lasers in dentistry and maxillofacial surgery, will continue to advance the application of Einstein's "splendid light" in their operations, to the benefit of patient and practitioner alike. For many procedures, lasers are now becoming the treatment of choice by both clinicians and patients, and in some cases, the standard of care [7]. Despite the common way of stimulated emission, that characterizes all laser emission, semiconductor laser are quite different from other types of lasers in both operating performance and pumping mechanism. Diode lasers emit light in the range of near infrared region to the visible red region. The main advantages of semiconductor laser are its compactness, simplicity and efficiency. They require very little auxiliary equipment and can be readily linked to optical fibers that able the diode laser to be used for medical purposes [8]. A frenum is an anatomic structure formed by a fold of mucous membrane, connective tissue, and sometimes muscle fibers. The most important of these are the labial frenae in the mid-line of the upper and lower jaw, the buccal frenae in the premolar region and the lingual frenum of the mandible. The frenum embryologically originates as remnant of the central cells of the vestibular lamina at the midsagittal area. The labial frenum connects the lip to the gingival tissue covering the alveolar periosteum of both maxilla and mandible. The size of the frenum varies among individuals, as does the location where the frenum inserts into the alveolar mucosa and/ or gingiva. The level of frenal attachments may vary from the 
height of the vestibule to the crest of the alveolar ridge and even to the incisal papilla area in the anterior maxilla. While the lingual frenum attaches the tongue to the floor of the mouth or to the alveolar mucosa of mandible. The frena under normal conditions do not have pathological consequences. However, in some cases they can present clinical problems, fundamentally of orthodontic, prosthetic, phonetic or periodontal nature $[9,10]$. The aims of this study was to apply and assess the clinical usefulness of the laserassisted frenectomy using diode laser $980 \mathrm{~nm}$.

\section{Materials and Methods}

This clinical study was carried out at Department of Maxillofacial Surgery, Ramadi Teaching Hospital, Anbar Health Directorate, Anbar Province, Iraq. A total of 25 patients including $16(64 \%)$ male and $9(36 \%)$ female with age range from 3 months to 18 years old with mean age 6.5 years old. The details of the laser assisted frenectomy procedures were explained verbally to patients and their parent and each patient (and/or parent) was given a written instructions and questionnaire sheet. All patients (and/or parent) signed an agreement informed consent for using laser in surgery. All patients were evaluated by clinical examinations and documented by digital photos and asked to fill the questionnaire sheet upon follow up visit and all patients were prepared for laser surgery and viral screen (HBsAg, HCV, and HIV) was requested. The Chirolas $20 \mathrm{~W}, 980 \mathrm{~nm}$ diode laser was used. All personnel participating in the surgery wore protective safety eyeglasses and masks. The same surgical technique was used for all patients, who were operated on under sedative analgesia using injectable midazolam $(5 \mathrm{mg} / \mathrm{ml})$ I.V. in a dose of (0.03-0.1) $\mathrm{mg} / \mathrm{Kg}$; the dose and dose range depend on individual patient response. Xylocaine Spray $10 \%$ was sprayed over and around operation site frequently. Diode laser in CW mode, Power of $10 \mathrm{~W}$, with a power density of $7961.78 \mathrm{~W} / \mathrm{cm}^{2}$ was used. For laser-assisted labial frenectomy; the upper lip was pulled upward by the assistant hands, then frenum was tightened. The incision was started with the frenum from the attached gingivae and interdental papilla on the labial surface between the central incisors extending upward from inner side of upper lip to the depth of vestibule ending in a rhomboidal raw area, separating the fibers from the periosteum. In some patients, there was a large space between the central incisors, so the inter-dental papilla between the incisors and the fibrous band between two maxillae had to be removed, until the periosteum was reached. At the end of the operation the edges and the raw area were irradiated (in defocused beam) for coagulating tissue and producing a denatured layer as a biological dressing. After end of laser exposure, the surgical site was wiped off with normal saline wet cotton roll (Figure $1 \mathrm{a}, \mathrm{b}, \mathrm{c}, \mathrm{d}$ and e). While for laser assisted lingual frenectomy; the tongue was retracted upwards and posteriorly. The frenum was grasped and tightened by placing the operator fingers (index \& middle) as close to the base of the tongue as possible and retract the ventrum of the tongue and for better visualization. The tip of fiber optic was hold in perpendicular or oblique direction to the frenum in contact mode between the laser tip and tissue surface. The procedure was completed by cutting first above and down avoiding the vessels and glands in the floor of the mouth. After end of laser exposure, the surgical

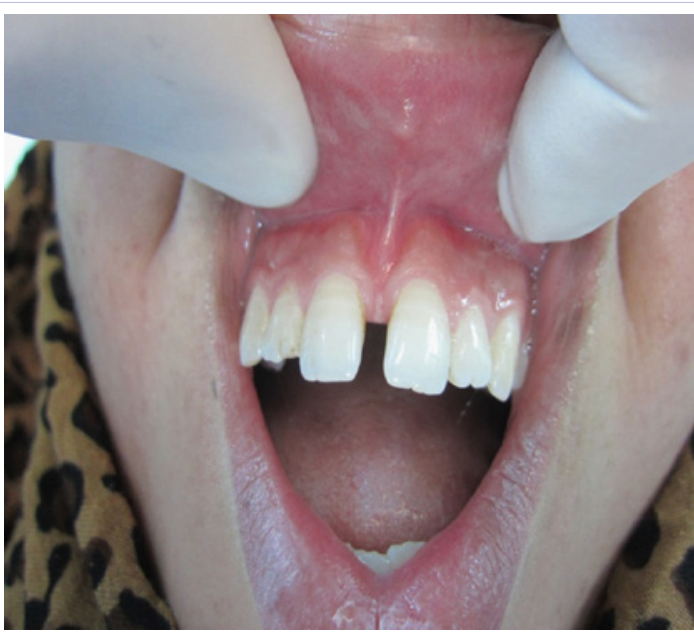

Figure 1a: Labial frenum.

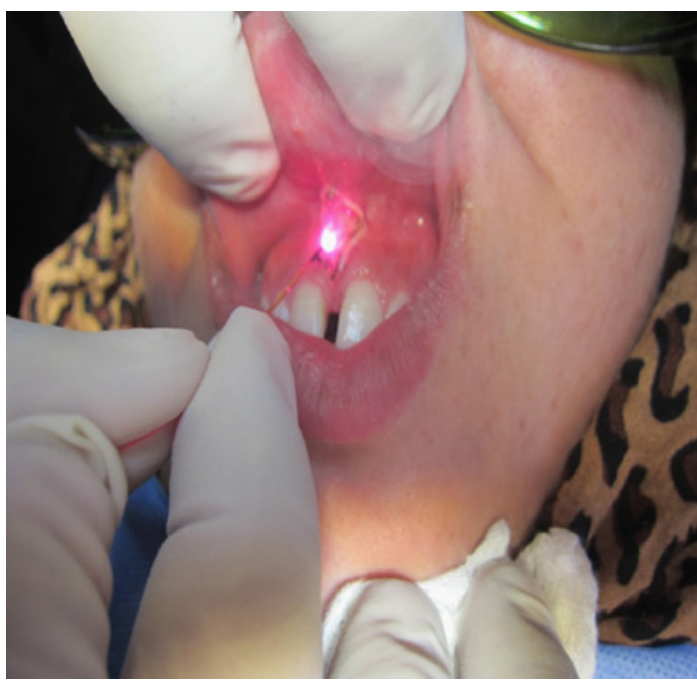

Figure 1b: Application of laser.

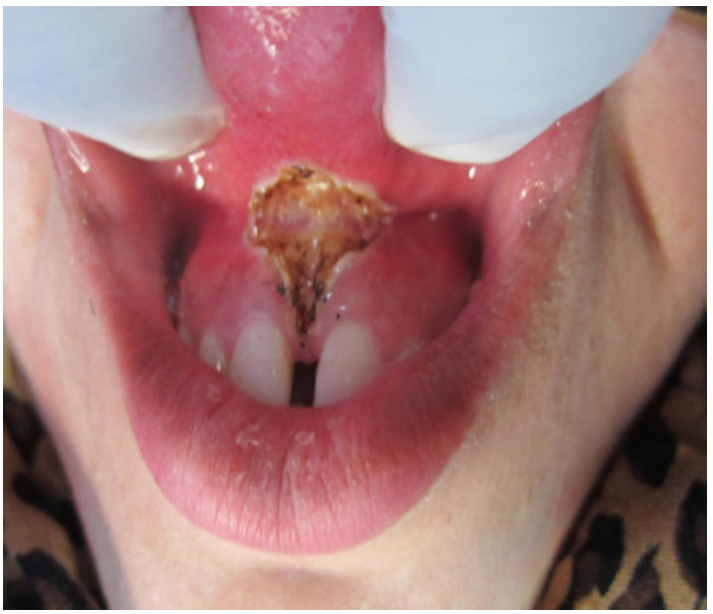

Figure 1c: Laser cutting. 


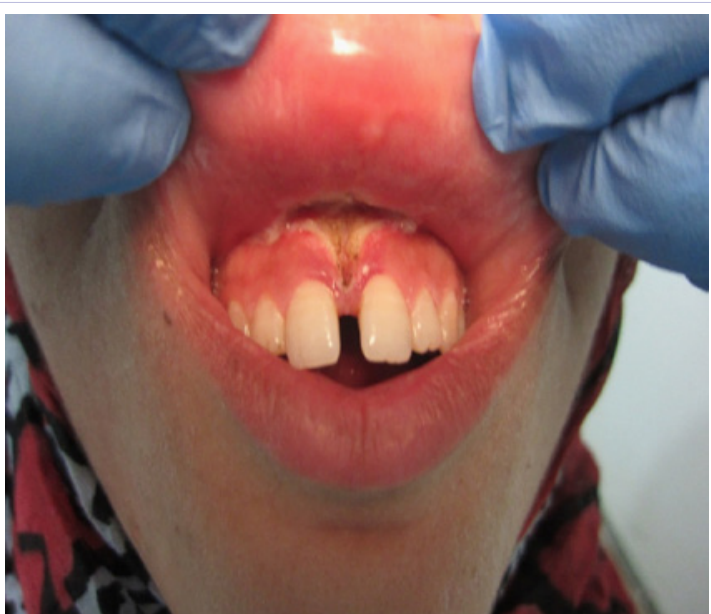

Figure 1d: End of Surgical operation.

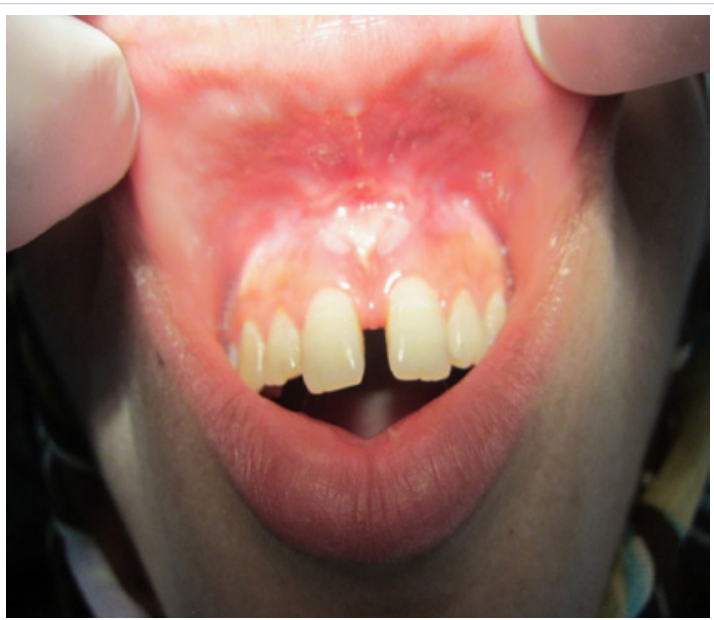

Figure 1e: End of treatment.

site was wiped off with normal saline wet cotton roll (Figure 2 a,b,c,d and e). After surgery, all the patients were given verbal instructions that including; avoid taking hot, spicy, citrus and hard foods for a few days, soft diet instructions, meticulous oral hygiene is practiced, patients were asked to fill in a questionnaire chart for the study. Because of local circumstances and crisis of Anbar province with restriction of hospital sterilization and to overcome any infection; Cephalexin (Capsules $250 \mathrm{mg}$; $500 \mathrm{mg}$ ), (Syrup $125 \mathrm{mg} / \mathrm{ml} ; 250 \mathrm{mg} / \mathrm{ml}$ ) were prescribed for 3 days, and Ibuprofen (Capsules $250 \mathrm{mg}$ ), (Suspension $20 \mathrm{mg} / \mathrm{ml}$ ) was used as analgesia (on need) during the postoperative period, practice the physio-exercises i.e. separate the lip from the gingival tissue by pulling up the lip and move the tongue upwards and laterally frequent times a day and commitment to follow up appointments in the exact date. All patients were examined at 3 days, 1 week, 2 week, and 4 week after surgery to assess pain, bleeding, edema, functions and overall satisfaction. In the follow-up appointments, Clinical observations, assessments and documentation by digital photos were done by the operator during examination. In addition to the data collected from the questionnaire sheets including the patient's notes at the operation day and during follow up visits.

\section{Results}

A total of 25 patients including 16 (64\%) male and 9 (36\%) female with an age range of 3 months to 18 years old with mean age 6.5 years old at Department of Maxillofacial Surgery, Ramadi Teaching Hospital, Anbar Health Directorate. The details of the laser assisted frenectomy procedures were explained verbally to patients. Patients was examined and evaluated clinically and prepared for surgery. Demographic and clinical data, including information concerning patient's age, sex, clinical presentation, past dental history, medical history were recorded (Figure 3). All the patients were undergone sedative analgesia and topical anesthesia spray during the surgical operation therefore no patient experience pain during the surgical operation and only $2(8 \%)$ patients experience mild pain during first three days postoperatively when postoperative pain was assessed clinically and by the patient self-assessment by subjected assessment of pain using the scale of $(0=$ no pain; $1=$ mild pain; $2=$ moderate

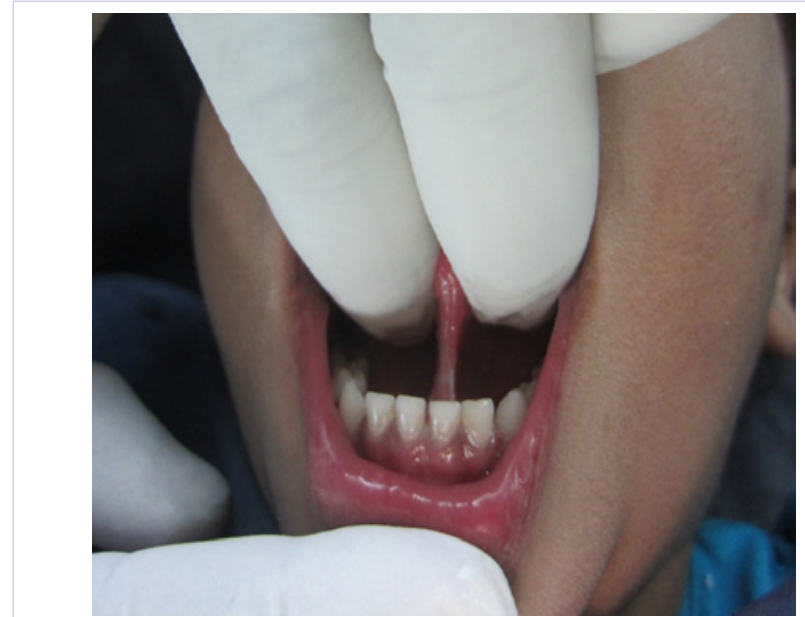

Figure 2a: Lingual frenum.

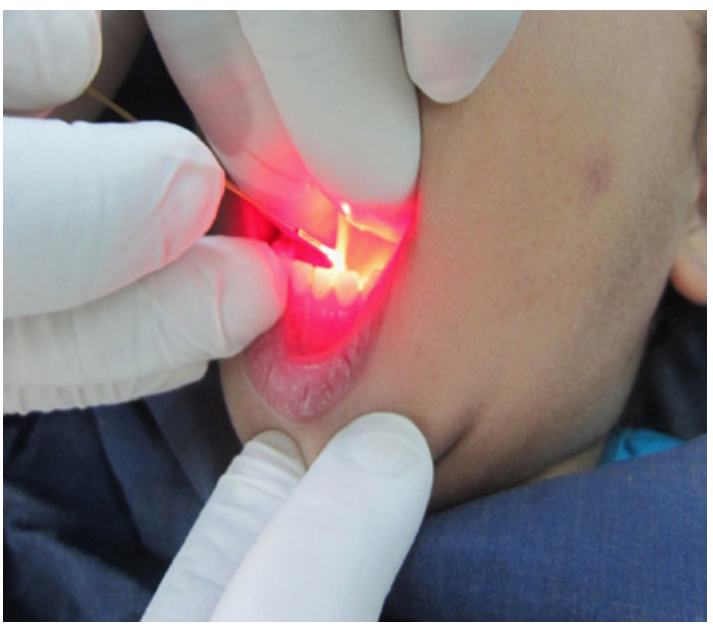

Figure 2b: Lingual frenum. 


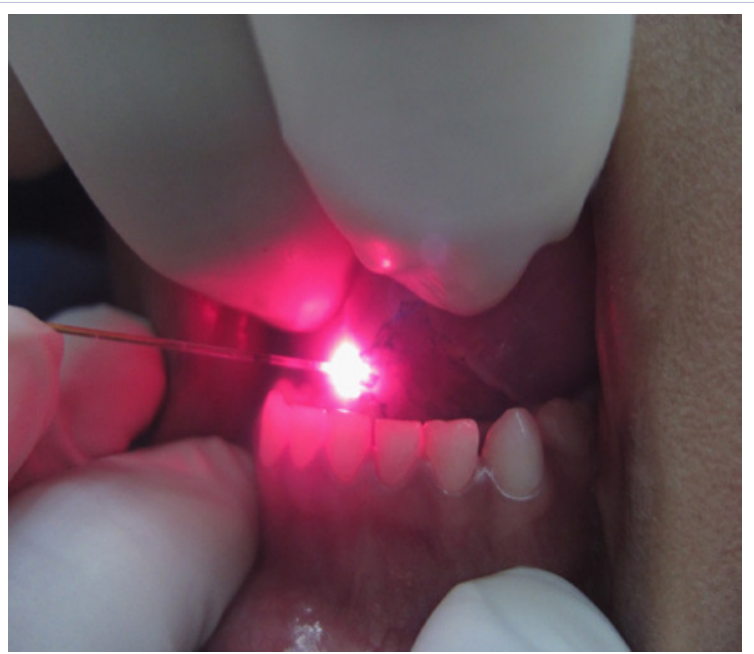

Figure 2c: Laser cutting.

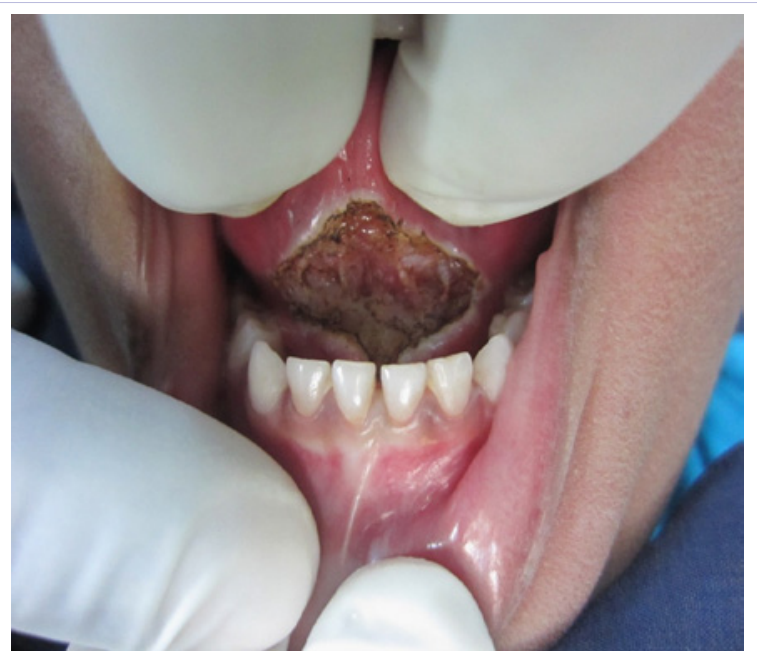

Figure 2d: End of Surgical operation.

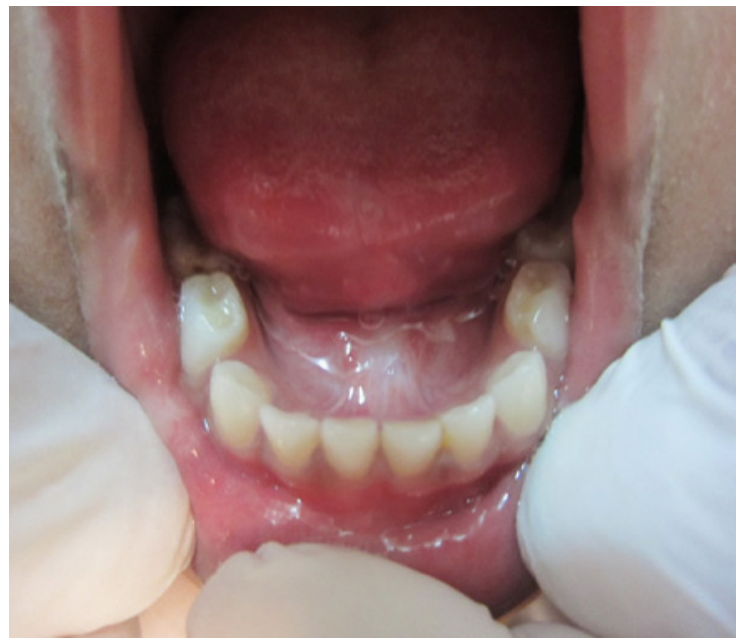

Figure 2e: End of Surgical operation. pain; and 3 = sever pain) and systemic analgesic usage as prescribed on need only; otherwise no patient experience pain during one week, two weeks and four weeks postoperatively. The intra-operative field was bloodless and there was no need to use sutures, after laser-assisted frenectomy, in all cases; which give us clear surgical field. In this clinical study, the surgical procedure was well tolerated by all the patients who were very co-operative during laser treatment. The wound was left open (to be healed by second intention) covered with coagulative layer which was clearly formed on the irradiated tissue surface, that effectively eliminated bleeding after the operation that was assessed using the scale of ( $0=$ no bleeding; $1=$ mild bleeding; 2 $=$ moderate bleeding; and $3=$ severe bleeding) . The coagulated tissue changed into a layer of scab tissue within 3 days, and the raw area was contracted from the margins of the surgical wound to its center gradually, closing inward until the wound surface was clinically healed within 10-14 days. Clinical assessment of the postoperative edema using the scale of $(0=$ no edema; $1=$ edema bleeding; 2 = moderate edema; and 3 = severe edema); there was mild edema (E/O and /or I/O) that noticed in 4 (16\%) during first three days after the surgery; then it subsided gradually, otherwise no patient showed edema during one week, two weeks and four weeks postoperatively. At each follow-up appointment, the operator was asking each patient to describe and rank their functional abilities disturbance that include speaking, eating, and brushing using scale of $(0=$ No function; 1 = mild function; 2 = moderate function; and 3 = sever function). Postoperative Functional abilities were within normal limit except in $4(16 \%)$ patients who was speaking with mild limitation in functional abilities with first three days postoperatively; e.g. painful eating, soreness during brushing, swallowing, while no patient revealed limited or disturbed functional abilities during one week, two weeks and four weeks postoperatively. In general patients were comfortable with no pain, either intra-operatively or post-operatively with fewer functional complications. Only 7 (28\%) revealed good (50-75\%) overall satisfaction and 18 (72\%) showed excellent (76-100\%) overall satisfaction during the

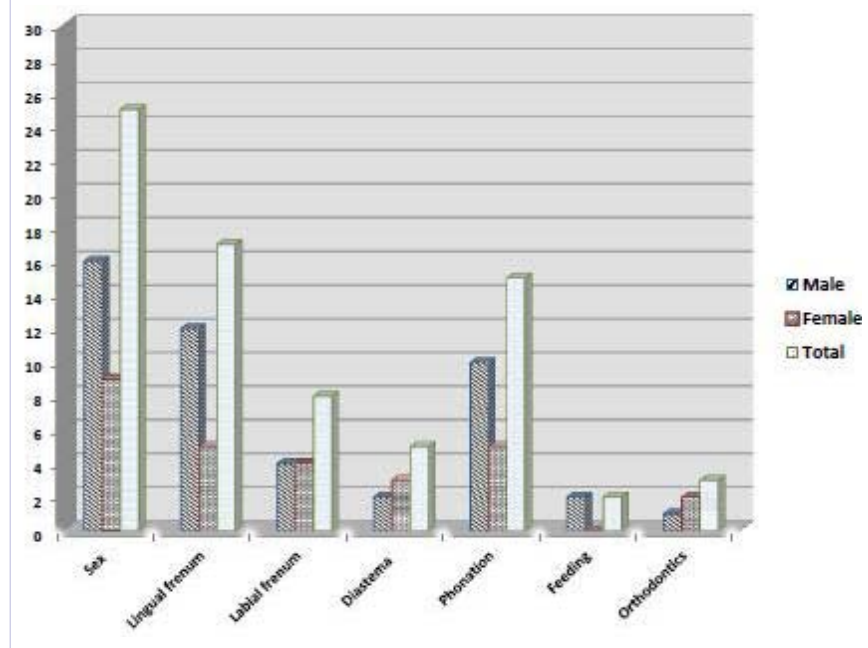

Figure 3: Patient Characteristics. 
first three days postoperatively, while all patients; 25 (100\%); showed marked excellent overall satisfaction throughout one week, two weeks and four weeks postoperatively .

\section{Discussion}

Photothermal interaction with tissue is the basic concept of surgical laser. In this process, radiant light is absorbed by the tissue and transformed to heat energy changing tissue structure. Laser light within was converted to thermal energy on contact with the tissue, causing laser tissue interaction, that when appropriately applied, can produce reaction ranging from incision, vaporization, to coagulation $[3,4]$. This wavelength has affinity for melanin or dark pigments, and is strongly absorbed by the blood hemoglobin, which contributes to their thermal effect. Therefore, this laser works more efficiently when the energy applied in the presence of pigments. This was the reason that homeostasis occurs with this wavelength. In the preliminary study, Diode laser creates a wound that can be characterized as thermal injury. The fiber was kept in steady motion and in contact mode that was used directly over the target tissue. The heated tip of optical fiber was used as a knife to create thermal effects. So keeping the fiber in one spot will gradually heat a growing mass of tissue in that spot and will produce a laser wound. The depth of penetration of the laser wound made by a contact probe will vary with the power used and duration of exposure time. Medical applications of lasers have focused particular interest on the interactions of infrared laser light in biological tissue. The physical heterogeneity of biological tissue results in considerable regional variation in light absorption and scattering, two of the critical processes that determine the pattern of heating. Since lasers were first introduced into dentistry, there have been investigations done to establish the laser parameters (i.e., wavelength, power density, continuous or pulsed mode, time of exposure, and spot size) that are most effective and least harmful for treating the soft and hard tissues of the oral cavity. Laser treatment has served as an alternative or adjunctive treatment to more conventional therapies because of its many advantages, including ablation or vaporization, homeostasis, and sterilization $[7,8]$. There are several factors related to tissue such as optical characteristics of the tissue, color, vascularity, consistency and thermal tissue properties that determine the effect of laser on the tissue. For routine clinical dental treatments, pain control is quite important for patient physical and mental well-being, as well as for the effectiveness of therapy. The laser a coagulation effect on small vessels that provides hemostasis and seals the sensory nerve endings, providing the needed anesthesia was reduced in this clinical series study $[11,12]$. Care was taken when multiple passes were necessary, using wet cotton roll and this was done in all the cases during the procedure. Almost none of the patients complained of pain during operations that agreed by Kato et al. [3,5]. In this present study, the use of $980 \mathrm{~nm}$ diode laser allowing increased surgical precision and accuracy, thereby reducing unnecessary damage to underlying tissues, and the procedure was with no bleeding in all cases, resulting in improving visualization of the surgical field, eliminating the need for postoperative sutures, and shortening the operation time, probably the efficiency of the laser allows sealing lymphatic and blood vessels that renders a bloodless surgical field [13-15] Studies. Hemostasis occurs because the generated heatcoagulates the tissue at the wound edges. Generally it is desirable to have no bleeding but it is also desirable to have the coagulated region as thin as possible. Hemostasis occurs because of denaturation of plasma proteins, hemoglobin and peri-vascular tissue locally. Also coagulation produces hemostasis by contraction of the vessel wall. This hemostasis may account for the minimal fluid extravasation observed, with a minimal inflammatory response around the tissues under surgical management. There was no need for sutures in all the cases because of the formed coagulated layer over the raw area with no evident bleeding, and to avoid the primary intention healing and reattachment $[12,14]$. In this clinical work, all patients were satisfied with good acceptance with respect to patient perceptions after laser and conventional surgical frenectomy and suggested that laser surgical frenectomy was superior to the conventional technique regarding patient perception and postsurgical discomfort. In addition, all patients returned to their normal day activities because of diminution of surgery time, depletion of blood loss and pain besides that patients were provided with minimally invasive surgery, where tissue injury is reduced to a minimal degree [3-5,16]. In this study, there was no post-surgical edema, because of laser-induced hemostasis and reduced tissue injury, except in three patients who had a mild edema that subsided after three days because of a relatively larger raw area, resulting in a more inflammatory response $[12,17,18]$, since it is the result of a reduced swelling and a minimal inflammatory response and this can be explained by precision surgical laser procedures without any adverse effect on surrounding normal tissue, and the ability of lasers to seal lymphatic channels results reduced postoperative edema, which in turn results in less postoperative discomfort, also the sealing of nerve endings resulting in reduced inflammatory response, and the formation of a fibrin clot over the surgical wound that protects the wound from external irritation, causing less pain after surgery and avoiding the use of analgesic drugs [11]. Patients treated with laser surgery have no functional complications since there was no damage to adjacent healthy tissues, with less wound contraction during healing; meaning that there is less mucosal scarring, resulting in satisfactory mobility of the soft tissue and consequently, there is a minimal oral dysfunction $[13,14,18,19]$. One of the main benefits of using dental lasers is the ability to interact selectively and precisely with diseased tissues, that explains the less degree of surrounding tissue injury, no significant complications, limited scarring and contraction and probably bio-stimulation effect of laser.

\section{References}

1. Carruth JAS. The principles of laser surgery. Scott Brown's otolaryngology. 5th edition Basic Sci. ch. 23, 1987.

2. Charschan SS. Laser in Industry. Western Electric Company, Incorporated, Van Norstrand Reinhold Company. 1972, p. 52-55.

3. Catone GA, Alling C. Laser Application in Oral and Maxillofacial Surgery. Philadelphia, W. B. Saunders Company, first edition, 1997. p. $30-40,115-146,181-193$.

4. Kishen Aand Asundi A. Fundamentals and Applications of Biophotonics 
in Dentistry, Series on Biomaterials and Bioengineering. Vol 4, London Imperial College Press, 2007. 123-151.

5. Markolf $\mathrm{H}$, Neimz. Laser-tissue interactions: fundamentals and applications, 3rd revised edition 2007 Springer Ch.1-3, 1-100.

6. Whinnery JR. Lasers Invention to Application. National Academy Press Washington, D.C, 1988. Ch.4, 100-106.

7. Baxter GD, Diamantopoulos C, O'Kane S, Shields T. D, Allen J. Therapeutic Lasers Theory and Practice. Harcourt Publishers Limited, first edition. pp. 56-64. 1999.

8. Miserendino LJ, Pick M. Laser in Dentistry. Quintessence publishing Co. pp. 35-54, 114-120. 1995.

9. Hashim ZM and Al-Fatlawi FA. Contributing Factors of Median Diastema in Iraqi Adults. M.Sc. thesis, department of POP, college of Dentistry, University of Baghdad 2006.

10. Kotlow L. Diagnosis and Treatment of ankyloglossia and tied maxillary fraenum in infants using Er: YAG and 1064 diode lasers. Eur Arch Paediatr Dent. 2011; 12(2):106-112.

11.Pie-Sanchez J, Espana-Tost AJ, Arnabat-Dominguez J, Gay-Escoda C Comparative study of upper lip frenectomy with the $\mathrm{CO} 2$ laser versus the Er, Cr:YSGG laser . Med Oral Patol Oral Cir Bucal. 2012; 17(2): 228232.

12. Tuncer I, Ozcakir-Tomruk C, Sencift K, and Çologlu S. Comparison of Conventional Surgery and CO2 Laser on Intraoral Soft Tissue Pathologies and Evaluation of The Collateral Thermal damage. Photomed Laser Surg. 2010; 28 (1): 75-79. doi: 10.1089/ pho.2008.2353.

13. White JM, Goodis HE, Rose CL. Use of the pulsed Nd:YAG laser for intraoral soft tissue surgery. Lasers Surg Med. 1991;11(5):455-61.

14. Kotlow LA: Using the Erbium: YAG Laser to correct abnormal lingual frenum attachments in newborns. Journal of the Academy of Laser Dentistry, 2004; 12(3):22-23.

15. Kotlow LA. Lasers in Pediatric Dentistry. Dent Clin North Am. 2004; 48(4): 889-922.

16. Kara C. Evaluation of patient perceptions of frenectomy: a comparison of Nd:YAG laser and conventional techniques. Photomed Laser Surg. 2008; 26(2):147-52. doi: 10.1089/pho.2007.2153.

17. Pick RM, Pecaro BC. Use of the CO2 Laser in Soft Tissue Dental Surgery. Lasers Surg Med. 1987; 7(2); 207-213.

18. Puthussery FJ, Shekar K, Gulati A, Downie IP. Use of carbon dioxide laser in lingual frenectomy. Br J Oral Maxillofac Surg. 2011; 49(7): 580-581. doi: 10.1016/j.bjoms.2010.07.010.

19. Fornaini C, Rocca JP, Bertrand MF, Merigo E, Nammour S, Vescovi P. Nd: YAG and Diode Laser in the Surgical Management of Soft Tissues Related to Orthodontic Treatment. Photomed Laser Surg. 2007; 25(5); 381- 92 . 